\title{
Diversity of the free-living marine and freshwater Copepoda (Crustacea) in Costa Rica: a review
}

\author{
Álvaro Morales-Ramírez 1,2, Eduardo Suárez-Morales ${ }^{3}$, \\ Marco Corrales-Ugalde ${ }^{4}$, Octavio Esquivel Garrote ${ }^{4}$
}

I Centro de Investigación en Ciencias del Mar y Limnología (CIMAR) 2 Escuela de Biología, Universidad de Costa Rica, 11501-2060 San José, Costa Rica 3 Colegio de la Frontera Sur (ECOSUR), Chetumal, Mexico 4 Licenciatura en Biología, Escuela de Biología, Universidad de Costa Rica, 11501-2060 San José, Costa Rica

Corresponding author: Álvaro Morales-Ramírez (alvaro.morales@ucr.ac.cr)

Academiceditor:I.S. Wehrtmann|Received 15December2013|Accepted18September2014|Published25November 2014

http://zoobank.org/C5A880B8-7598-4C10-8A26-97D9D99320EB

Citation: Morales-Ramírez A, Suárez-Morales E, Corrales-Ugalde M, Garrote OE (2014) Diversity of the free-living marine and freshwater Copepoda (Crustacea) in Costa Rica: a review. In: Wehrtmann IS, Bauer RT (Eds) Proceedings of the Summer Meeting of the Crustacean Society and the Latin American Association of Carcinology, July Costa Rica, 2013. ZooKeys 457: 15-33. doi: 10.3897/zookeys.457.6820

\begin{abstract}
The studies on marine copepods of Costa Rica started in the 1990's and focused on the largest coastalestuarine systems in the country, particularly along the Pacific coast. Diversity is widely variable among these systems: 40 species have been recorded in the Culebra Bay influenced by upwelling, northern Pacific coast, only 12 in the Gulf of Nicoya estuarine system, and 38 in Golfo Dulce, an anoxic basin in the southern Pacific coast of the country. Freshwater environments of Costa Rica are known to harbor a moderate diversity of continental copepods ( 25 species), which includes 6 calanoids, 17 cyclopoids and only two harpacticoids. Of the +100 freshwater species recorded in Central America, six are known only from Costa Rica, and one appears to be endemic to this country. The freshwater copepod fauna of Costa Rica is clearly the best known in Central America. Overall, six of the 10 orders of Copepoda are reported from Costa Rica. A previous summary by 2001 of the free-living copepod diversity in the country included 80 marine species (67 pelagic, 13 benthic). By 2009, the number of marine species increased to 209: 164 from the Pacific (49\% of the copepod fauna from the Eastern Tropical Pacific) and 45 from the Caribbean coast ( $8 \%$ of species known from the Caribbean Basin). Both the Caribbean and Pacific species lists are growing. Additional collections of copepods at Cocos Island, an oceanic island $530 \mathrm{~km}$ away of the Pacific coast, have revealed many new records, including five new marine species from Costa Rica. Currently, the known diversity of marine copepods of Costa Rica is still in development and represents up to $52.6 \%$ of the total marine microcrustaceans recorded in the country. Future sampling and taxonomic efforts in the
\end{abstract}

Copyright Álvaro Morales-Ramírez et al. This is an open access article distributed under the terms of the Creative Commons Attribution License (CC BY 4.0), which permits unrestricted use, distribution, and reproduction in any medium, provided the original author and source are credited. 
marine habitats should emphasize oceanic environments including deep waters but also littoral communities. Several Costa Rican records of freshwater copepods are likely to represent undescribed species. Also, the biogeographic relevance of the inland copepod fauna of Costa Rica requires more detailed surveys.

\section{Keywords}

New records, biodiversity, Pacific, Caribbean, microcrustaceans, biogeography

\section{Introduction}

Copepods are a highly diverse group of crustaceans; over 13000 species of this subclass have been described (Boxshall and Halsey 2004, Boxshall and Defaye 2008) and many more are added each year. Copepods are also one of the most abundant metazoans in the world. Up to $60-80 \%$ of the marine zooplankton biomass in neritic and oceanic environments is represented by copepods (Casanova et al. 1982). This abundance is related to the success of the planktonic forms in colonizing the oceanic water column (Boxshall and Halsey 2004). Huys and Boxshall (1991) summarized the economic and biological importance of copepods.

The free-living forms inhabit a wide variety of aquatic environments including also those with extreme conditions of salinity and temperature. They are common members of the biotas of subterranean waters (Pipan 2005), anchialine systems (SuárezMorales and Iliffe 2005), hypersaline waters (Brucet et al. 2009), and bromeliads, among others (Reid 1986).

Although the importance of the free-living copepods is clear, information on their diversity and distribution is scarce and scattered in many regions. In the case of marine forms, Mauchline (1998) listed 13 Large Marine Ecosystems (LME) that have some data regarding their copepod fauna, but information on most of the remaining $75 \%$ of LMEs remains largely incomplete. Similarly, the freshwater environments harbor a diverse copepod fauna; in general, the knowledge of the group in the Americas is asymmetrical, Central America being the least studied subregion in the continent. As part of the Neotropical region with a high continental copepod diversity (Boxshall and Defaye 2008), studies of the group in Costa Rica have been intermittent (Collado et al. 1984a, b, Suárez-Morales and Reid, unpubl. data), but new efforts are revealing interesting records (Suárez-Morales et al. 2011).

A periodical revision of the progress of the knowledge of the copepod fauna in Costa Rica is a key tool to evaluate their potential diversity in marine and freshwater environments. It is also useful to detect invasive species in different aquatic habitats, which is a worldwide phenomenon representing a serious threat to the aquatic biodiversity (Molnar et al. 2008). The present paper summarizes the information about copepods that has been recorded until now in Costa Rica, a country with two coastlines and with a high habitat diversity. 


\section{Methods}

\section{Surveyed aquatic systems in Costa Rica}

The main coastal and oceanic environments and also the lakes and freshwater sites in which copepods have been surveyed in Costa Rica are presented in Figure 1. These areas and systems were selected because of their physiographic or ecological features: the northern Pacific coast is influenced by the Gulf of Papagayo jetdriven upwelling system (Mc Creary et al. 1989, Chelton et al. 2000). The Gulf of Nicoya, located in the central Pacific Costa Rican coast is one of the largest and well-studied tropical estuaries, with a surface area of $1530 \mathrm{~km}^{2}$ (Vargas 1995), and Golfo Dulce to the south is an anoxic fjord-like embayment (Svendsen et al. 2006). The Cocos Island is the only emergent point of an oceanic submarine ridge (Protti et al. 2012), situated $496 \mathrm{~km}$ off Cabo Blanco, Pacific coast, with a important marine diversity (Cortés 2012), and since 1997 a UNESCO World Heritage Site (Morales-Ramírez 2008). The Caribbean coast is represented mainly by river deltas dominated by waves and barrier beaches to the north, and sandy beaches alternating with a few formations of coral reef fossils to the south (Denyer and Cárdenas 2000). The most studied freshwater bodies are Lake Arenal, studied since the 1980's, and smaller lakes like Cote and Fraijanes (Collado et al. 1984a, b, Umańa and Collado 1990).

We reviewed literature records of free-living marine, brackish water, and freshwater copepods found in Costa Rica. Up to thirty seven sites have been sampled for copepods in the country, including marine and freshwater environments (Fig. 1). Marine pelagic copepods were sampled using different gears including WP-2 standard zooplankton nets with mesh sizes of 100, 150, 200, and $500 \mu \mathrm{m}$. Vertical tows were performed at depths less than $100 \mathrm{~m}$ in Golfo Dulce and Cocos Island (Morales-Ramírez and Nowaczyk 2006, Quesada-Alpízar and Morales Ramírez 2006, Morales-Ramírez 2008). The same kind of tows were used to sample copepods in the Gulf of Papagayo (Bednarski and Morales-Ramírez 2004, Rodríguez-Saénz and Morales-Ramírez 2012), Gulf of Nicoya and Coronado Bay (Morales-Ramírez 1996). In the Caribbean Sea, organisms were collected mostly with horizontal surface tows (Morales-Ramírez and Murillo 1996, Carrillo 2012). For marine benthic copepods, sampling techniques were described by Mielke (1992, 1994 a,b,c). This community was also sampled by collecting sediment samples with cores from beaches (Mielke 1992, 1994a, b, c, 1995), but also by a van Veen grab sampler (Sibaja-Cordero 2012). Freshwater copepods were obtained by different devices but mainly by nets used in different habitats including littoral and limnetic areas of the water bodies (see Collado et al. 1984a, b, Suárez-Morales and Reid, unpubl. data). 


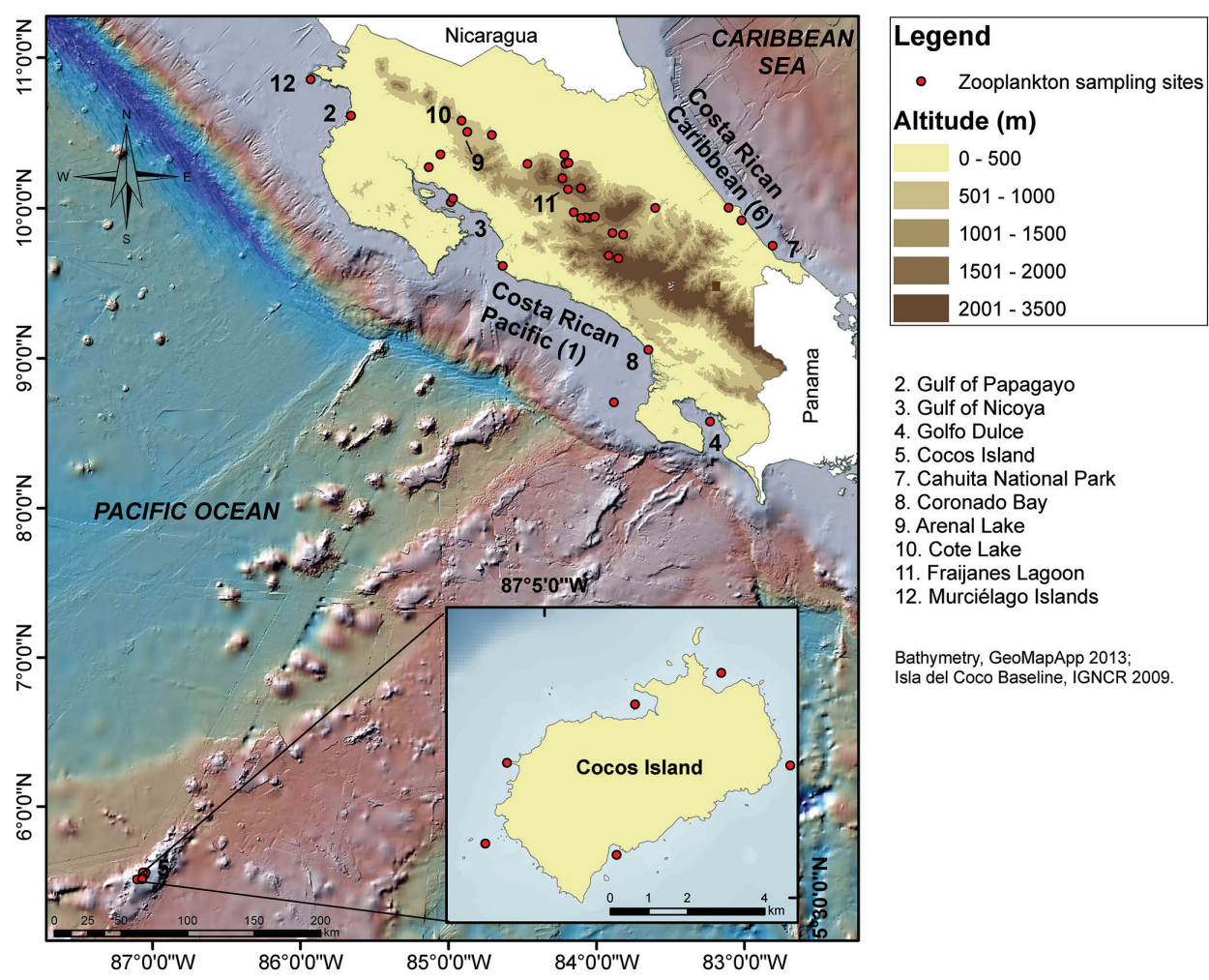

Figure I. Maps with the sampling sites for marine and freshwater copepods in Costa Rica.

\section{Results and discussion}

\section{Historical overview}

\section{Marine pelagic copepods}

The copepod species found in marine systems of Costa Rica are listed in Table 1. The zooplankton of the Pacific coast of Costa Rica has been surveyed more intensely and during a longer period of time than in the Caribbean side. The three sectors (northern, central and southern) of the Pacific coast include interesting systems in which the copepod fauna has been studied. In Culebra Bay, an embayment associated to the Gulf of Papagayo (north Pacific coast), Bednarski (2001) recorded 39 species. Suárez-Morales and Morales-Ramírez (2001) reported the calanoid Acartia negligens Dana, 1849 near Murciélago Islands at the northern Pacific coast of Costa Rica and expanded its known regional range. In the same region, a new species (Cymbasoma concepcionae SuárezMorales and Morales-Ramírez 2003) of the order Monstrilloida was described; it was the first record of monstrilloids in Costa Rica. Also in the northern Pacific sector, an oceanic upwelling system known as the Costa Rica Dome has been the subject of many zooplankton studies because of its importance as a fisheries region (Fiedler 2002). 
Table I. List of Copepoda found in marine environments of Costa Rica. Records from published works of the Costa Rican marine copepod fauna. Localities in Costa Rica: 1 - Costa Rican Pacific, 2 - Gulf of Papagayo, 3 - Gulf of Nicoya, 4 - Golfo Dulce, 5 - Cocos Island, 6 - Costa Rican Caribbean, 7 - Cahuita National Park. References: 1. Morales-Ramírez and Suárez-Morales (2009), 2. Carrillo (2012), 3. SibajaCordero (2012), 4. Morales-Ramírez et al. (unpubl. data), 5. Morales-Ramírez and Vargas (1995), 6. Suárez-Morales et al. (2013), 7. Suárez-Morales and Morales-Ramírez (2009), 8. Morales-Ramírez (2001).

\begin{tabular}{|c|c|c|c|}
\hline Family & Species & Locality & References \\
\hline \multicolumn{4}{|c|}{ Gymnoplea } \\
\hline \multicolumn{4}{|c|}{ Order Calanoida } \\
\hline \multirow{4}{*}{ Acartiidae } & Acartia clausi Giesbrecht, 1889 & $1,3,4,5$ & 1,4 \\
\hline & Acartia danae Giesbrect, 1889 & $1,3,4,5$ & 1,4 \\
\hline & Acartia lilljeborgii Giesbrecht, 1889 & $1,2,3,4,5$ & 1,4 \\
\hline & Acartia negligens Dana, 1849 & 2 & 1 \\
\hline \multirow{12}{*}{ Aetideidae } & Aetideopsis rostrata G.O. Sars, 1905 & 1 & 1 \\
\hline & Aetideus armatus Boeck, 1872 & 1 & 1 \\
\hline & Aetideus giesbrechti (Cleve, 1904) & 5 & 4 \\
\hline & Chiridius Giesbrecht, 1892 & 1 & 1 \\
\hline & Euchirella brevis G.O. Sars, 1905 & 1 & 1 \\
\hline & Euchirella rostrata Claus, 1866. & 7 & 1 \\
\hline & Euaetideus giesbrechti Sars, 1925 & 1 & 1 \\
\hline & Gaetanus brevispinus Sars, 1903 & 1 & 1 \\
\hline & Gaetanus miles Giesbrecht, 1888 & 1 & 1 \\
\hline & Gaetanus minor Farran, 1905 & 1 & 1 \\
\hline & Gaidius tenuispinus Sars, 1900 & 1 & 1 \\
\hline & Valdiviella brevicornis Steuer, 1904 & 1 & 1 \\
\hline Arietellidae & Arietellus sp. Giesbrecht, 1892 & 1 & 1 \\
\hline \multirow{7}{*}{ Augaptilidae } & Augaptilus longicaudatus Giesbrecht, 1889 & 1 & 1 \\
\hline & Augaptilus megalurus Giesbrecht, 1889 & 7 & 2 \\
\hline & Haloptilus acutifrons Giesbrecht, 1892 & 1 & 1 \\
\hline & Haloptilus longicornis Claus, 1863 & 1,6 & 1 \\
\hline & Haloptilus mucronatus Claus, 1863 & 1 & 1 \\
\hline & Haloptilus ornatus Giesbrecht, 1892 & 1 & 1 \\
\hline & Haloptilus oxycephalus Giesbrecht, 1889 & 1 & 1 \\
\hline \multirow{9}{*}{ Calanidae } & Neocalanus cristatus (Krøyer, 1848) & 1 & 1 \\
\hline & Calanus pacificus Brodsky, 1948 & 5 & 4 \\
\hline & Mesocalanus tenuicornis (Dana, 1849) & 1 & 1 \\
\hline & Canthocalanus pauper (Giesbrecht, 1888) & $1,2,3,4,5$ & 1,4 \\
\hline & Cosmocalanus darwini (Lubbock, 1860) & 1,5 & 1,4 \\
\hline & Nannocalanus minor Claus, 1863 & $1,3,4,5$ & 1,4 \\
\hline & Neocalanus gracilis Dana, 1849 & $1,2,5$ & 1,4 \\
\hline & Neocalanus robustior (Giesbrecht, 1888) & 1,5 & 1,4 \\
\hline & Undinula vulgaris Dana, 1842 & $\begin{array}{l}1,2,3,4 \\
5,6,7\end{array}$ & $1,2,4$ \\
\hline \multirow{5}{*}{ Calocalanidae } & Calocalanus pavo Dana, 1849 & $1,5,7$ & $1,2,4$ \\
\hline & Calocalanus pavoninus Farran, 1926 & 1 & 1 \\
\hline & Calocalanus styliremis Giesbrecht, 1888 & $1,3,4$ & 1 \\
\hline & Calocalanus contractus Farran, 1926 & 1 & 1 \\
\hline & Ishnocalanus plumulosus Claus, 1863 & 1 & 1 \\
\hline Candaciidae & Candacia catula Giesbrecht, 1881 & $1,2,3,5$ & 1,4 \\
\hline
\end{tabular}




\begin{tabular}{|c|c|c|c|}
\hline Family & Species & Locality & References \\
\hline & Candacia truncata Dana, 1846 & 1,5 & 1,4 \\
\hline & Candacia pachydactyla Dana, 1849 & 1,5 & 1,4 \\
\hline & Candacia varicans Giesbrecht, 1892 & 1 & 1 \\
\hline \multirow{6}{*}{ Centropagidae } & Centropages abdominalis (Sato, 1913) & 5 & 4 \\
\hline & Centropages bradyi (Wheeler, 1900) & 5 & 4 \\
\hline & Centropages calaninus (Dana, 1849) & 1,5 & 1,4 \\
\hline & Centropages furcatus (Dana, 1849) & 1,5 & 1,4 \\
\hline & Centropages gracilis (Dana, 1849) & 5 & 4 \\
\hline & Centropages longicornis Mori, 1932 & 1,5 & 1,4 \\
\hline \multirow{3}{*}{ Clausocalanidae } & Clausocalanus arcuicornis (Dana, 1849) & 1,5 & 1,4 \\
\hline & Clausocalanus furcatus Brady, 1883 & 1,5 & $1,2,4$ \\
\hline & Clausocalanus pergens Farran, 1926 & $1,3,4$ & 1 \\
\hline \multirow{13}{*}{ Eucalanidae } & Eucalanus attenuatus Dana, 1849 & 1,5 & 1,4 \\
\hline & Eucalanus bungii Giesbrecht, 1892 & 1 & 1 \\
\hline & Eucalanus crassus (Giesbrecht, 1888) & 7 & 2 \\
\hline & Eucalanus elongatus Dana, 1849 & 1 & 1 \\
\hline & Eucalanus inermis Griesbrecht, 1892 & 1 & 1 \\
\hline & Eucalanus monachus Giesbrecht, 1888 & 6 & 1 \\
\hline & Eucalanus mucronatus Giesbrecht, 1891 & 1 & 1 \\
\hline & Eucalanus pileatus Giesbrecht, 1888 & 1 & 1 \\
\hline & Eucalanus sewelli Fleminger, 1973 & 1,5 & 1,4 \\
\hline & Eucalanus subcrassus Giesbrecht, 1888 & 5,6 & $1,2,4$ \\
\hline & Eucalanus subtenuis Giesbrecht, 1888. & 1,5 & 1,4 \\
\hline & Rhincalanus cornutus Dana, 1849. & 5,6 & 1,4 \\
\hline & Rhincalanus nasutus Giesbrecht, 1888 & 1,5 & 1,4 \\
\hline \multirow{13}{*}{ Euchaetidae } & Euchaeta acuta Giesbrecht, 1892 & 1 & 1 \\
\hline & Euchaeta plana Philippi, 1843 & 5 & 4 \\
\hline & Euchaeta barbata Brady, 1883 & 1 & 1 \\
\hline & Euchaeta indica Wolfenden, 1905 & 1,5 & 1,4 \\
\hline & Euchaeta longicornis Giesbrecht, 1888 & 1,5 & 1,4 \\
\hline & Euchaeta marina (Prestandrea, 1833) & 1,5 & 1,4 \\
\hline & Euchaeta media Giesbrecht, 1888 & 1 & 1 \\
\hline & Euchaeta rimana (Bradford, 1974) & 5 & 4 \\
\hline & Euchaeta tenuis Esterly, 1906 & 1 & 1 \\
\hline & Euchaeta wolfendeni Scott, 1909 & 1 & 1 \\
\hline & Paraeuchaeta hansenii (With, 1915) & 1 & 1 \\
\hline & Paraeuchaeta norvegica (Boeck, 1872) & 1 & 1 \\
\hline & Paraeuchaeta tonsa (Giesbrecht, 1895) & 1 & 1 \\
\hline \multirow{2}{*}{ Heterorhabdidae } & Heterorhabdus papilliger Claus, 1863 & 1 & 1 \\
\hline & Scaphocalanus sp. G.O. Sars, 1900 & 1 & 1 \\
\hline \multirow{5}{*}{ Lucicutiidae } & Lucicutia bicornuta Wolfenden, 1911 & 1 & 1 \\
\hline & Lucicutia flavicornis Claus, 1963 & $1,5,6$ & 1,4 \\
\hline & Lucicutia gemina Farran, 1926 & 1 & 1 \\
\hline & Lucicutia grandis Giesbrecht, 1895 & 1 & 1 \\
\hline & Lucicutia ovalis Giesbrecht, 1889 & 1 & 1 \\
\hline Mecynoceridae & Mecynocera clausi Thompson, 1888 & 1,5 & 1,4 \\
\hline \multirow{2}{*}{ Metridinidae } & Metridia sp. Boeck, 1864 & 1 & 1 \\
\hline & Pleuromamma abdominalis f. edentata Steuer, 1931 & 1 & 1 \\
\hline
\end{tabular}




\begin{tabular}{|c|c|c|c|}
\hline Family & Species & Locality & Reference \\
\hline & Pleuromamma abdominalis f. abyssalis Steuer, 1931 & 1 & 1 \\
\hline & Pleuromamma abdominalis abdominalis Lubbock, 1856 & 1 & 1 \\
\hline & Pleuromamma gracilis Claus, 1863 & 1,5 & 1,4 \\
\hline & Pleuromamma piseki Farran, 1929 & 1 & 1 \\
\hline & Pleuromamma quadrungulata Dahl, 1893 & 1 & 1 \\
\hline & Pleuromamma robusta Dahl, 1893 & 1 & 1 \\
\hline & Pleuromamma scutullata Brodsky, 1950 & 1 & 1 \\
\hline & Pleuromamma xiphias (Giesbrecht, 1889) & 1 & 1 \\
\hline \multirow{6}{*}{ Paracalanidae } & Acrocalanus gibber Giesbrecht, 1888 & $1,3,4,5$ & 1,4 \\
\hline & Acrocalanus gracilis Giesbrecht, 1888 & 1,5 & 1,4 \\
\hline & Acrocalanus longicornis Giesbrecht, 1888 & 1 & 1,2 \\
\hline & Paracalanus aculeatus Giesbrecht 1888 & 1,5 & 1,2 \\
\hline & Parvocalanus crassirostris Dahl, 1894 & 1 & 1 \\
\hline & Paracalanus parvus Claus, 1863 & 1 & 1 \\
\hline \multirow{3}{*}{ Phaennidae } & Cephalophanes sp. Sars, 1907 & 1 & 1 \\
\hline & Cornucalanus sp. Wolfenden, 1905 & 1 & 1 \\
\hline & Phaenna spinifera Claus, 1863 & 1,5 & 1,4 \\
\hline \multirow{11}{*}{ Pontellidae } & Calanopia americana F. Dahl, 1894 & $1,5,6$ & 1,4 \\
\hline & Labidocera acuta Dana, 1849 & 1,5 & 1,4 \\
\hline & Labidocera aestiva Wheeler, 1901 & $1,5,7$ & $1,2,4$ \\
\hline & Labidocera dentruncata (Dana, 1849) & 5 & 4 \\
\hline & Labidocera lubboki Giesbrecht, 1892 & 1 & 1 \\
\hline & Labidocera scotti Giesbrecht, 1897 & 7 & 1,2 \\
\hline & Pontella agassizii Giesbrecht, 1895 & 1 & 1 \\
\hline & Pontella mimocerami Fleminger 1957 & 6 & 1 \\
\hline & Pontellina plumata Dana, 1849 & 1,5 & 1,4 \\
\hline & Pontellopsis villosa Brady, 1883 & 1 & 1 \\
\hline & Pontellopsis yumadae (Mori 1937) & 5 & 4 \\
\hline \multirow{5}{*}{ Pseudodiaptomidae } & Pseudodiaptomus acutus Dahl, 1894 & 6 & 1 \\
\hline & Pseudodiaptomus cristobalensis Marsh, 1919 & 1 & 1 \\
\hline & Pseudodiaptomus marshi Wright, 1936 & 6 & 1 \\
\hline & Pseudodiaptomus panamensis Walter, 1989 & 3 & 8 \\
\hline & Pseudodiaptomus wrigthi Johnson, 1964 & 1 & 1 \\
\hline \multirow{9}{*}{ Scolecithricidae } & Amallothrix gracilis Sars, 1925 & 1 & 1 \\
\hline & Lophothrix sp. Giesbrecht, 1895 & 1 & 1 \\
\hline & Scolecithricella dentata (Giesbrecht, 1892) & 1 & 1 \\
\hline & Scolecithricella marginata (Giesbrecht, 1888) & $1,3,4$ & 1 \\
\hline & Scolecithricella tenuiserrata (Giesbrecht, 1892) & 1 & 1 \\
\hline & Scolecithricella vittata (Giesbrecht, 1892) & 1 & 1 \\
\hline & Scolecithricella bradyi (Giesbrecht, 1888) & 1,5 & 1 \\
\hline & Scolecithrix danae Lubbock, 1856 & $1,5,6$ & 1,4 \\
\hline & Scottocalanus sp. Sars, 1905 & 1 & 1 \\
\hline \multirow{5}{*}{ Temoridae } & Eurytemora Giesbrecht, 1881 & 1 & 1 \\
\hline & Temora discaudata Giesbrecht, 1889 & 1,5 & 1,4 \\
\hline & Temoropia mayumbaensis Scott, 1894 & $1,3,4,5$ & 1,4 \\
\hline & Temora stylifera (Dana, 1849) & 5,7 & 2,4 \\
\hline & Temora turbinata Dana, 1849 & 1 & 1,2 \\
\hline
\end{tabular}




\begin{tabular}{|c|c|c|c|}
\hline Family & Species & Locality & Reference \\
\hline \multicolumn{4}{|c|}{ Super Order PODOPLEA } \\
\hline \multicolumn{4}{|c|}{$\begin{array}{l}\text { Order CYCLOPOIDA } \\
\end{array}$} \\
\hline \multirow{14}{*}{ Corycaeidae } & Corycaeus bremhi Dana, 1849 & 1 & 1 \\
\hline & Corycaeus catus Dana, 1845 & 5 & 4 \\
\hline & Corycaeus clausi Dahl F., 1894 & 7 & 2 \\
\hline & Corycaeus crassiusculus (Dana, 1848) & 5 & 4 \\
\hline & Corycaeus (Agetus) flaccus Giesbrecht, 1891 & $1,3,4$ & 1 \\
\hline & Corycaeus furcifer (Claus, 1863) & 5 & 4 \\
\hline & Corycaeus latus (Dana, 1848) & 5 & 4 \\
\hline & Corycaeus limbatus Brady, 1883 & 7 & 2 \\
\hline & Corycaeus (Corycaeus) speciosus Dana, 1849 & $1,5,7$ & $1,2,4$ \\
\hline & Corycaeus robustus (Giesbrecht, 1891) & 5 & 4 \\
\hline & Corycaeus (Onychocorycaeus) ovalis Claus, 1863 & 1,5 & 1,4 \\
\hline & Farranula carinata Giesbrecht, 1891 & 5 & 4 \\
\hline & Farranula gibbula Giesbrecht, 1981 & 1,5 & 1,4 \\
\hline & Farranula gracilis Dana, 1849 & 6 & 1 \\
\hline \multirow{6}{*}{ Oithonidae } & Oithona hebes Giesbrecht, 1891 & 6 & 1 \\
\hline & Oithona nana Giesbrecht, 1893 & 6 & 2 \\
\hline & Oithona plumifera Bair, 1843 & $1,5,6$ & 1,4 \\
\hline & Oithona setigera Claus, 1863 & 1,6 & 1,2 \\
\hline & Oithona similis Claus, 1863 & $1,5,6$ & 1,4 \\
\hline & Oithona spinirostris Claus, 1863 & 1 & 1 \\
\hline \multirow{7}{*}{ Oncaeidae } & Conaea gracilis Dana, 1853 & 1,6 & 1 \\
\hline & Lubbockia aculeata Giesbrecht, 1892 & 1 & 1 \\
\hline & Oncaea conifera Giesbrecht, 1891 & 1,5 & 1,4 \\
\hline & Oncaea mediterranea Claus, 1883 & 1,5 & 1,4 \\
\hline & Oncaea ornata Giesbrecht, 1891 & 1 & 1 \\
\hline & Oncaea venusta Phillippi, 1843 & $1,5,7$ & 1,4 \\
\hline & Pachos punctatum (Claus, 1863) & 5 & 4 \\
\hline Clausidiidae & Hemicyclops thalassius Vervboort \& Ramírez, 1966 & 3 & 5 \\
\hline \multirow{13}{*}{ Sapphirinidae } & Copilia longistylis (Mori, 1932) & 5 & 4 \\
\hline & Copilia mirabilis Dana, 1852 & 5,7 & 2,4 \\
\hline & Copilia quadrata (Dana, 1852) & 5 & 4 \\
\hline & Copilia vitrea Haeckel, 1864 & 1,5 & 1,4 \\
\hline & Sapphirina angusta (Dana, 1849) & 5 & 4 \\
\hline & Sapphirina darwinii (Haeckel, 1864) & 5 & 4 \\
\hline & Sapphirina gastrica (Giesbrecht, 1891) & 5 & 4 \\
\hline & Sapphirina metallina (Dana, 1849) & 5 & 4 \\
\hline & Sapphirina nigromaculata Claus, 1863 & 1,5 & 1,2 \\
\hline & Sapphirina opalina Dana, 1849 & 1,5 & 1,4 \\
\hline & Sapphirina ovatolanceolata Dana, 1852 & 1,5 & 1,4 \\
\hline & Sapphirina scarlata Giesbrecht, 1891 & 1,5 & 1,4 \\
\hline & Vettoria sp. Wilson C.B., 1924 & & \\
\hline \multicolumn{4}{|c|}{ Order MONSTRILLOIDA } \\
\hline \multirow{3}{*}{ Monstrillidae } & Cymbasoma alvaroi Suárez-Morales \& Carrillo, 2013 & 7 & 6 \\
\hline & Cymbasoma cocoense Suárez-Morales \& Morales-Ramírez, 2009 & 5 & 7 \\
\hline & $\begin{array}{l}\text { Cymbasoma concepcionae Suárez-Morales \& Morales-Ramírez, } \\
2003\end{array}$ & 1 & 1 \\
\hline
\end{tabular}




\begin{tabular}{|c|c|c|c|}
\hline Family & Species & Locality & References \\
\hline & Monstrilla grandis Giesbrecht, 1891 & 7 & 6 \\
\hline & $\begin{array}{l}\text { Monstrillopsis cahuitae Suárez-Morales, Carrillo \& Morales- } \\
\text { Ramírez, } 2013\end{array}$ & 7 & 6 \\
\hline & $\begin{array}{l}\text { Monstrillopsis chathamensis Suárez-Morales \& Morales-Ramírez, } \\
2009\end{array}$ & 5 & 7 \\
\hline \multicolumn{4}{|c|}{ Order MORMONILLOIDA } \\
\hline \multirow{2}{*}{ Mormonillidae } & Mormonilla minor Giesbrecht, 1891 & 1 & 1 \\
\hline & Mormonilla phasma Giesbrecht, 1891 & 1 & 1 \\
\hline \multicolumn{4}{|c|}{$\begin{array}{l}\text { Order } \\
\text { HARPACTICOIDA }\end{array}$} \\
\hline Aegisthidae & Aegistus aculeatus Giesbrecht, 1891 & 6 & 1 \\
\hline Canuellidae & Microcanuella bisetosa Mielke, 1994 & 1 & 1 \\
\hline Cletodidae & Cletodes sp. Brady, 1872 & 5 & 3 \\
\hline \multirow{2}{*}{ Clytemnestridae } & Clytemnestra rostrata Brady, 1883 & 1,5 & $1,2,4$ \\
\hline & Clytemnestra scutellata Dana, 1847 & 1,5 & $1,2,4$ \\
\hline \multirow{3}{*}{ Ectinosomatidae } & Halectinosoma sp. Vervoot, 1962 & 5 & 3 \\
\hline & Microsetella cf. norvegica (Boeck, 1865) & 5 & 3 \\
\hline & Microsetella rosea Dana, 1848 & 1,5 & 1,4 \\
\hline \multirow{5}{*}{ Diosaccidae } & Balucopsylla triarticulata Wells \& Rao, 1987 & 1 & 1 \\
\hline & Schizopera nicoyana Mielke, 1995 & 3 & 1 \\
\hline & Schizopera osana Mielke, 1995 & 1 & 1 \\
\hline & Schizopera sp. A Mielke, 1995 & 1 & 1 \\
\hline & Schizopera sp. B Mielke, 1995 & 1 & 1 \\
\hline Harpacticidae & Zausodes septimus Lang, 1965 & 1 & 1 \\
\hline \multirow{4}{*}{ Laophontidae } & Afrolaophonte schmidti Mielke, 1997 & 1 & 1 \\
\hline & Klienychocamptoides itoi Mileke, 1981 & 1 & 1 \\
\hline & Laophontella horrida dentata Por, 1964 & 1 & 1 \\
\hline & Mexicolaophonte arganoi Cottarelli, 1977 & 1 & 1 \\
\hline Leptastacidae & Leptastacidae undet. & 5 & 3 \\
\hline Longipediidae & Longipedia helgolandica Klie, 1949 & 6 & 1 \\
\hline \multirow{5}{*}{ Miraciidae } & Amonardia sp. Lang, 1944 & 5 & 3 \\
\hline & Amphiascopsis cf. cinctus (Claus, 1866) & 5 & 3 \\
\hline & Macrosetella gracilis Dana, 1852 & 1,5 & $1,2,4$ \\
\hline & Robertgurneya sp. Lang, 1944 & 5 & 3 \\
\hline & Typhlamphiascus sp. Lang, 1944 & 5 & 3 \\
\hline Orthopsyllidae & Orthopsyllus linearis curvaspina Claus, 1886 & 5 & 3 \\
\hline Paramesochridae & Paramesochridae indet. & 5 & 3 \\
\hline Peltidiidae & Peltidium nichollsi Geddes, 1968 & 6 & 2 \\
\hline \multirow{4}{*}{ Phyllopodidae } & Phyllopodopsyllus ancylus Mielke, 1992 & 1 & 1 \\
\hline & Phyllopodopsyllus carinatus Mielke, 1992 & 1 & 1 \\
\hline & Phyllopodopsyllus gertrudi costaricensis Kunz, 1984 & 1 & 1 \\
\hline & Phyllopodopsyllus setouchiensis Kitazima, 1981 & 1 & 1 \\
\hline Euterpinidae & Euterpina acutifrons (Dana, 1847) & $1,5,6$ & 1 \\
\hline Thalestridae & Amenophia sp. Boeck, 1865 & 5 & 2 \\
\hline Tetragonicipitidae & Oniscopsis robinsoni Chappuis \& Delamare, 1956 & 6 & 1 \\
\hline
\end{tabular}




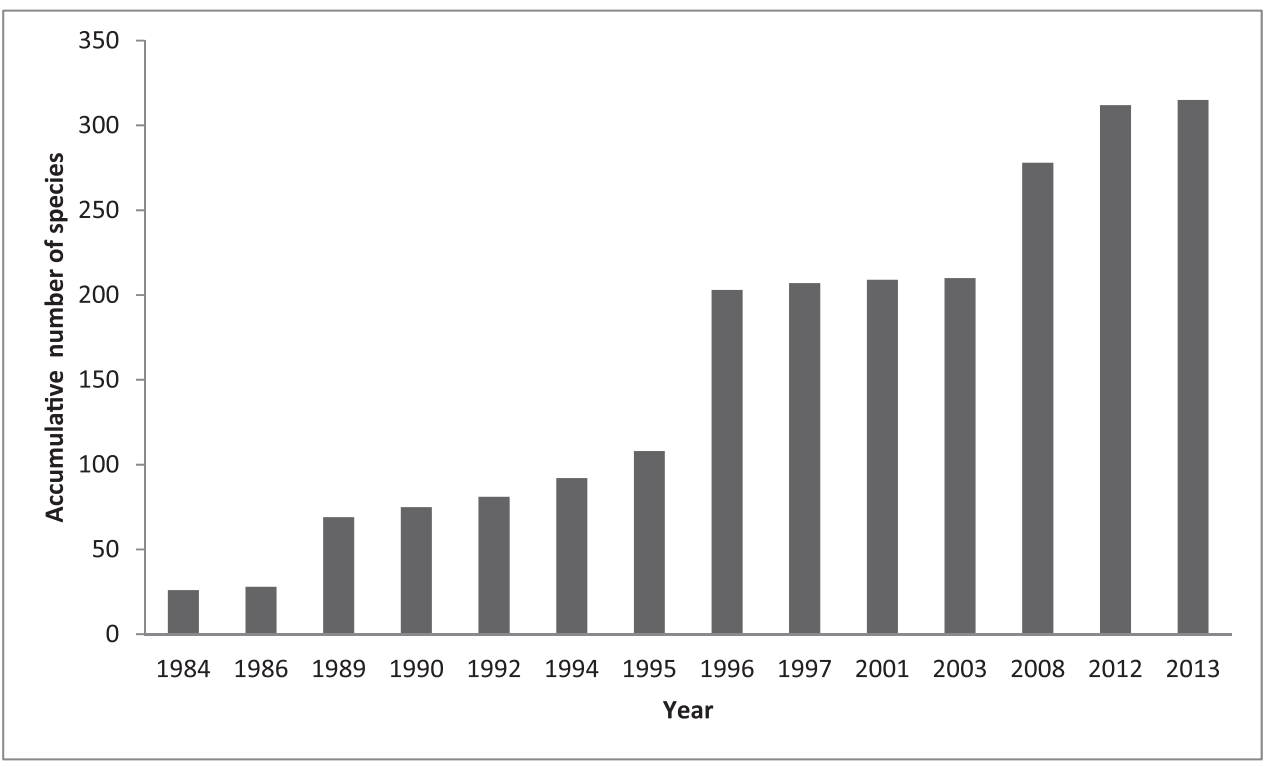

Figure 2. Accumulative number of species of marine and freshwater copepods recorded per year in Costa Rica. Data from the revision of the published literature.

A total of 41 copepod species have been recorded in surface waters of this highly productive area (Suárez-Morales and Gasca 1989, Fernández-Alamo and Vicencio 1996).

In the Gulf of Nicoya, an estuarine system on the central Pacific coast of Costa Rica, Morales-Ramírez and Vargas (1995) reported 12 copepod species dwelling at the inner sector of the gulf. They determined $A$. lilljeborgii and members of the family Pseudodiaptomidae as the most abundant taxa. Further studies in the Gulf of Nicoya raised the total number of species to 32 (Morales-Ramírez 1996).

Zooplankton studies in the southern Pacific area of Costa Rica have focused on two locations. The first one is Coronado Bay, which is part of the Térraba-Sierpe mangrove system. Its copepod fauna comprised 13 species of a few families, mainly Clausocalanidae, Calanidae and Paracalanidae (Morales-Ramírez 1996). The second location is Golfo Dulce, where Morales-Ramírez (1996) recorded 38 species. The genera Corycaeus, Clausocalanus, Oncaea and Oithona were the most common in this area. This gulf harbors $21.5 \%$ of the marine diversity recorded from the Costa Rican Pacific coast (Morales-Ramírez 2011).

In the Caribbean coast of Costa Rica, studies on copepods are scarcer and more recent. In the Cahuita National Park, Morales-Ramírez (2001) collected 22 species, with $A$. lilljeborgii and species of the family Pontellidae as the dominant forms. At that time, 18 of those species were reported as new records for the Caribbean coast of Costa Rican. Further studies revealed 13 additional records (Carrillo 2012) and two new species of monstrilloid copepods: Monstrillopsis cahuitae Suárez-Morales, Carrillo \& Morales-Ramírez, 2013 and C. alvaroi Suárez-Morales \& Carrillo, 2013 (Suárez-Morales et al. 2013).

The zooplankton sampling efforts in Costa Rica have been carried out since the the 1980 's. Figure 2 shows the accumulative number of species progressively found by 
national investigators. There seems to be major contributions to a sustained increase since 1984, nevertheless, the period between 1996 and 2003 added few or none new records; subsequently, with the beginning of studies around Cocos Island National Park increased the number of records. The first studies that included some analysis of copepods as a group (not on a species level) were from the coastal area around the Cahuita National Park, a Caribbean reef system (Morales 1987), and Caño Island at the Pacific coast (Guzmán and Obando 1988). Considering these early surveys, it is concluded that the current knowledge of the copepod fauna comprises more than two decades (Fig. 2). In 2009, the list of species recorded in Costa Rica included 209 species of marine copepods: 185 were planktonic and 24 benthic, 165 in the Pacific coast and 44 in the Caribbean waters. These numbers excluded species from Cocos Island (Morales-Ramírez and Suárez-Morales 2009).

Periodical sampling of the zooplankton of Cocos Island started in 2007, with a 6 -station sampling plan around the island and in coral reefs patches. These samples first yielded 68 species of copepods (Morales-Ramírez 2008), a list that now includes 95 species (Table 1), and 14 new records of planktonic copepods, eight benthic forms (Morales-Ramírez et al. unpubl. data), and two new monstrilloids species: $C$. cocoense Suárez-Morales \& Morales-Ramírez, 2009 and M. chathamensis Suárez-Morales \& Morales-Ramírez, 2009; the last case represented the first record of the genus Monstrillopsis for Costa Rican waters (Suárez-Morales and Morales-Ramírez 2009). The species composition of the copepod fauna from Cocos Island and adjacent oceanic waters has been summarized by Morales-Ramírez (2008). The two most diverse families are Corycaeidae and Sapphirinidae (unpubl. data).

\section{Marine benthic copepods}

The number of free-living copepod taxa that have been described from material obtained in Costa Rica is summarized in Table 2. Taxonomic surveys of the benthic copepod fauna are scarce. Mielke (1992) analyzed beach sediments from locations along both coasts of Costa Rica: along the Caribbean coast, samples were obtained at three locations (Portete, Cahuita and Manzanillo beaches), whereas at the Pacific coast, samples were obtained from 13 sites. Six copepod taxa belonging to the family Tetragonicipitidae were found, including two new species (Phyllopodopsyllus ancylus Mielke, 1992 and P. carinatus Mielke, 1992) and two new subspecies (Laophontella horrida dentata Mielke, 1992 and P. gertrudi costaricensis Mielke, 1992). The monotypic genus Microcanuella and its species M. bisetosa Mielke, 1994 were described from sandy beaches of the Gulf of Nicoya. Mielke (1995) also found four species of Schizopera Sars G.O., 1905 and described Schizopera osana Mielke, 1995.

At Cocos Island, Sibaja-Cordero (2012) analyzed subtidal sediment samples and identified seven families, nine genera and three species of harpacticoid copepods. The family Miraciidae included four genera, one species and an undetermined taxon. This material is expected to reveal many additional species records of the Harpacticoida. 
Table 2. New species described from type material collected in Costa Rica. References. 1. Mielke (1994), 2. Mielke (1992), 3. Mielke (1994c), 4. Mielke (1995), 5. Suárez-Morales and Morales-Ramírez (2003), 6. Suárez-Morales and Morales-Ramírez (2009), 7. Suárez-Morales et al. (2013), 8. Suárez-Morales and Gasca (2012).

\begin{tabular}{|c|c|c|c|c|}
\hline Order & Species & Location & Environment & References \\
\hline \multirow[t]{10}{*}{ Harpacticoida } & Karllangia obscura Mielke, 1994 & $\begin{array}{c}\text { Manzanillo, } \\
\text { Caribbean coast }\end{array}$ & Mud flat & 1 \\
\hline & Karllangia pulchra Mielke, 1994 & $\begin{array}{l}\text { Manzanillo, } \\
\text { Caribbean coast }\end{array}$ & Mud flat & 1 \\
\hline & Phyllopodopsyllus carinatus Mielke, 1992 & $\begin{array}{c}\text { Manzanillo, } \\
\text { Caribbean coast }\end{array}$ & Beach slope & 2 \\
\hline & $\begin{array}{l}\text { Phyllopodopsyllus gertrudi costaricensis Mielke, } \\
1992\end{array}$ & $\begin{array}{c}\text { Manzanillo, } \\
\text { Caribbean coast }\end{array}$ & $\begin{array}{c}\text { Beach slope } \\
\text { and mangrove }\end{array}$ & 2 \\
\hline & Phyllopodopsyllus ancylus Mielke, 1992 & $\begin{array}{l}\text { Punta Morales, } \\
\text { Pacific coast }\end{array}$ & Mud flat & 2 \\
\hline & Orthopsyllus linearis curvaspina (Claus, 1886) & Pacific coast & Sandy beach & 3 \\
\hline & Microcanuella bisetosa Mielke, 1994 & $\begin{array}{l}\text { Gulf of Nicoya, } \\
\text { Pacific coast }\end{array}$ & Sandy beach & 3 \\
\hline & Schizopera nicoyana Mielke, 1995 & $\begin{array}{l}\text { Gulf of Nicoya, } \\
\text { Pacific coast }\end{array}$ & Sandy beach & 4 \\
\hline & Schizopera osana Mielke, 1995 & $\begin{array}{l}\text { Gulf of Nicoya, } \\
\text { Pacific coast }\end{array}$ & Sandy beach & 4 \\
\hline & Laophontella horrida dentata (Por, 1964) & Pacific coast & Sandy beach & 2 \\
\hline \multirow[t]{5}{*}{ Monstrilloida } & $\begin{array}{c}\text { Cymbasoma concepcionae Suárez-Morales \& } \\
\text { Morales-Ramírez, } 2003\end{array}$ & $\begin{array}{c}\text { Bahía Culebra, } \\
\text { Pacific coast }\end{array}$ & pelagic & 5 \\
\hline & $\begin{array}{c}\text { Cymbasoma cocoense Suárez-Morales \& Morales- } \\
\text { Ramírez, } 2009\end{array}$ & $\begin{array}{l}\text { Cocos Island, } \\
\text { Pacific Ocean }\end{array}$ & pelagic & 6 \\
\hline & $\begin{array}{c}\text { Monstrillopsis chathamensis Suárez-Morales \& } \\
\text { Morales-Ramírez, } 2009\end{array}$ & $\begin{array}{l}\text { Cocos Island, } \\
\text { Pacific coast }\end{array}$ & pelagic & 6 \\
\hline & $\begin{array}{l}\text { Monstrillopsis cahuitae Suárez-Morales, Carrillo } \\
\text { \& Morales-Ramírez, } 2013\end{array}$ & $\begin{array}{l}\text { Cahuita National } \\
\text { Park, Caribbean } \\
\text { coast }\end{array}$ & pelagic & 7 \\
\hline & $\begin{array}{c}\text { Cymbasoma alvaroi Suárez-Morales, Carrillo \& } \\
\text { Morales-Ramírez, } 2013\end{array}$ & $\begin{array}{l}\text { Cahuita National } \\
\text { Park, Caribbean } \\
\text { coast }\end{array}$ & pelagic & 7 \\
\hline Siphonostomatoida & $\begin{array}{l}\text { Lepeophtheirus alvaroi Suárez-Morales \& } \\
\text { Gasca, } 2012\end{array}$ & $\begin{array}{l}\text { Cocos Island, } \\
\text { Pacific Ocean }\end{array}$ & water column & 8 \\
\hline
\end{tabular}

\section{Freshwater copepods}

The freshwater zooplankton of Costa Rica has been studied since the 1980's, but these surveys have been intermittent (Collado et al. 1984a, b, Dussart and Fernando 1986, Reid 1990a, Umaña and Collado 1990, Haberyan et al. 1995). Since then, a total of 25 species of copepods have been recorded (Table 3). Cyclopid copepods are the most diverse and abundant group in inland waters of Costa Rica as found in other Neotropical areas (Suárez-Morales et al. 2000, Silva 2008). Diaptomid copepods, the most representative group of the order Calanoida in freshwater environments, show a low diver- 
Table 3. Species of freshwater copepods recorded in Costa Rica (* new range extension includes Costa Rica, ${ }^{* *}$ only known from Costa Rica). References: 1. Collado et al. (1984a), 2. Collado et al. (1984b), 3. Dussat and Fernando (1986), 4. Reid (1990a), 5. Reid (1990b), 6. Gavlas (2012), 7. Suárez-Morales et al. (2013).

\begin{tabular}{|c|c|c|}
\hline Family & Species & References \\
\hline \multicolumn{3}{|l|}{ Gymnoplea } \\
\hline \multicolumn{3}{|l|}{ Order Calanoida } \\
\hline \multirow{3}{*}{ Diaptomidae } & Arctodiaptomus dorsalis (Marsh, 1907) & 2 \\
\hline & Diaptomus sp. Westwood, 1836 & 1 \\
\hline & Prionodiaptomus colombiensis (Thiébaud, 1912)* & 6 \\
\hline \multicolumn{3}{|c|}{ Super Order PODOPLEA } \\
\hline \multicolumn{3}{|c|}{ Order CYCLOPOIDA } \\
\hline \multirow{20}{*}{ Cyclopidae } & Ectocyclops pharelatus (Koch, 1838) & 1 \\
\hline & Eucyclops agilis (Koch, 1838) & 1 \\
\hline & Eucyclops bondi Kiefer, 1934 & 2 \\
\hline & Eucyclops leptacanthus Kiefer, 1956 & 2 \\
\hline & Eucyclops serrulatus (Fischer, 1851) & 1 \\
\hline & Halicyclops exiguus Kiefer, 1934 & 1 \\
\hline & Macroyclops albidus (Jurine, 1820) & 1 \\
\hline & Mesocyclops brasilianus Kiefer, 1933 & 1 \\
\hline & Mesocyclops leuckarti (Claus, 1857) & 1 \\
\hline & Mesocyclops thermocyclopoides Harada, 1931 & 2,7 \\
\hline & Microcyclops ceibaensis (Marsh, 1919) & 2 \\
\hline & Microcyclops dubitabilis Kiefer, 1934 & 2 \\
\hline & Microcyclops varicans (G.O Sars, 1863) & 1 \\
\hline & Paracyclops fimbriatus (Fischer, 1853) & 2 \\
\hline & Thermocyclops crassus (Fischer, 1853) & 2 \\
\hline & Thermocyclops decipiens (Kiefer, 1929) & 2 \\
\hline & Thermocyclops inversus (Kiefer, 1936) & 2 \\
\hline & Thermocyclops tenuis (Marsh, 1910) & 2 \\
\hline & Tropocyclops prasinus (Fishcer, 1860) & 2 \\
\hline & Tropocyclops pseudoparvus Dussart \& Fernando, 1986** & 3 \\
\hline \multicolumn{3}{|c|}{ Order HARPACTICOIDA } \\
\hline \multirow{2}{*}{ Canthocamptidae } & Attheyella fubrmanni (Thiébaud, 1912) & 4 \\
\hline & Canthocamptus striblingi (Reid, 1990) ${ }^{* *}$ & 5 \\
\hline
\end{tabular}

sity in Costa Rica, a feature which is also shared with Central America (Suárez-Morales 2003, Suárez-Morales et al. 2005). Their diversity could be underestimated in Costa Rica; records of presumedly cosmopolitan species like Eucyclops agilis, E. serrulatus could include undescribed species (Alekseev et al. 2006, Gutiérrez-Aguirre et al. 2013). The Costa Rican E. bondi probably represents an undescribed species (Mercado-Salas and Suárez-Morales 2014). 


\section{General diversity}

Studies focusing on the taxonomic composition of the free-living copepod fauna have been carried out in selected locations involving coastal, oceanic and large continental aquatic systems (i.e., lakes as: Arenal, Cote, Bonilla, Fraijanes, and Cerro Chato). Currently, there are 281 species of copepods recorded in Costa Rica, representing 6 orders and 50 families. The order with the highest number of species is Calanoida (147 spp.), followed by Cyclopoida (73) and Harpacticoida (36). There are no records of species of the orders Gelyelloida, Platycopioida and Misophrioida.

According to our results, copepods are the most studied marine group of microcrustaceans in Costa Rica, representing almost $44 \%$ of the 473 marine species reported in the country until 2009 (Wehrtmann and Cortés 2009); this figure increases to $52.6 \%$ when studies around Cocos Island and Caribbean Sea as well new records from other areas are considered.

\section{Remarks}

\section{Marine copepods}

Being situated in a fully tropical area with the influence of both the Atlantic and Pacific oceans, the marine copepod fauna of Costa Rica is expected to be highly diverse. The analysis of the diversity is also an important tool to reveal changes and patterns of the pelagic communities surveyed. In these coastal systems, particularly along the Pacific coast, the diversity of copepods has detectable variations as a response to local oceanographic conditions. Also, typical upwelling species have been recorded in Culebra Bay, Gulf of Papagayo, with major seasonal changes in dominance as a response to upwelling conditions (Bednarski and Morales-Ramírez 2004). The Gulf of Nicoya is known to show an assemblage of typical estuarine species, where small calanoids dominate year-round (Brugnoli et al. 2004). The Golfo Dulce represents a mixed environment in which oceanic and coastal copepod species coexist, a condition that is intensified during El Niño events (Quesada-Alpízar and Morales-Ramírez 2006). These and other Costa Rican systems should be surveyed during different seasons and hydrographic conditions in order to develop a complete overview of their copepod diversity. Also, increased efforts should be carried out to explore the diversity of benthic copepods, mainly of harpacticoids, a group whose diversity in marine and freshwater environments is still poorly known in Costa Rica.

\section{Freshwater copepods}

Currently, 25\% of Central American records of freshwater species are from Costa Rica. Together with that of the other areas of Central America (CA), its inland copepod 
fauna represents an interesting assemblage, because CA is a recent biogeographic passage of copepods between North and South America (Suárez-Morales 2003, SuárezMorales et al. 2005). Also, there are records of introduced species of copepods in Costa Rica (Collado et al. 1984a, b, Suárez-Morales et al. 2011); their advancement in the country should be followed based on an expanded sampling program. Additional efforts are required to reveal the copepod diversity of Costa Rican freshwater systems. New inland aquatic habitats should be sampled, like ephemeral pools, subterranean waters, caves, methane seeps, and even semi-terrestrial habitats, often harboring a rich copepod fauna (Reid 1986).

A consistent plan to develop more human resources formed in the taxonomy and systematics of these microcrustaceans is required as a basic strategy to increase the knowledge of this biodiversity. Thereby we could increase the knowledge of our biological diversity and thus allow the development of improved conservation strategies (Mercado-Salas et al. 2013) and prevent or mitigate some of the problems related to the loss of biodiversity (Molnar et al. 2008).

\section{Acknowledgements}

We are grateful to two anonymous reviewers for their constructive comments on a previous version of the manuscript. Our gratitude to many field assistants, and to the Research Vice-Rectory of the University of Costa Rica for financial support through projects 808-94-252, 111-96-248, 808-96-601, 808-97-253, 808-A2-052, 808-A3062, 808-A7-401, 808-A7-116, 808-B0-060 granted to the first author.

\section{References}

Alekseev V, Dumont HJ, Pensaert J, Baribwegure D, Vanfleteren JR (2006) A redescription of Eucyclops (Fischer, 1851) (Crustacea: Copepoda: Cyclopoida) and some related taxa, with a phylogeny of the E. serrulatus-group. Zoologica Scripta 35: 123-147. doi: $10.1111 /$ j.1463-6409.2006.00223.x

Bednarski M (2001) Macrozooplankton of Culebra Bay, Costa Rica, with an emphasis on copepods. M.Sc. thesis, University of Bremen, Bremen, Germany.

Bednarski M, Morales-Ramírez A (2004) Composition, abundance and distribution of macrozooplankton in Culebra Bay, Gulf of Papagayo, Pacific coast of Costa Rica and its value as bioindicator of pollution. Revista de Biología Tropical 52(2): 105-118

Boxshall GA, Defaye D (2008) Global diversity of copepods (Crustacea: Copepoda) in freshwater. Hydrobiologia 595: 195-207. doi: 10.1007/s10750-007-9014-4

Boxshall GA, Halsey S (2004) An Introduction to Copepod Diversity. The Ray Society, London, 966 pp.

Brucet S, Boix D, Gascon S, Sala J, Quintana XD, Badosa A, Søndergaard M, Lauridsen TL, Jeppesen E (2009) Species richness of crustacean zooplankton and trophic structure of 
brackish lagoons in contrasting climate zones: north temperate Denmark and Mediterranean Catalonia (Spain). Ecography 32: 692-702. doi: 10.1111/j.1600-0587.2009.05823.x

Brugnoli-Olivera E, Díaz-Ferguson E, Delfino-Machin M, Morales-Ramírez A (2004) Composition of the zooplankton community, with emphasis in copepods, in the Punta Morales zone, Golfo de Nicoya, Costa Rica. Revista de Biología Tropical 52: 897-902

Carrillo A (2012) Diversidad, abundancia, composición y biomasa del zooplancton de la zona arrecifal del Parque Nacional Cahuita, Limón ¿Cuál es la disponibilidad de larvas de invertebrados bénticos 25 años después? Bachelor thesis, Universidad de Costa Rica, San José, Costa Rica.

Casanova J, Casanova F, Dueret F, Rampal J (1982) Biomasse et composition chimique et faunistique du zooplankton du sector senegambien (Campagne CINECA de la "Thalasse", aout 1975). Rapports et procès-verbaux des réunions / Conseil International pour l'exploration de la Mer 180: 266-269.

Chelton DB, Freilich MH, Esbensen SK (2000) Satellite observations of the wind jets off the Pacific coast of Central America. Part I: case studies and statistical characteristics. Monthly Weather Review 128: 1993-2018 doi: 10.1175/1520-0493(2000)128<1993:SOOTWJ>2.0.CO;2

Collado C, Fernando CH, Sephton D (1984a) The freshwater zooplankton of Central America and the Caribbean. Hydrobiologia 113: 105-119. doi: 10.1007/BF00026597

Collado C, Defaye D, Dussart B, Fernando CH (1984b) A study of freshwater Copepoda (Crustacea) of Costa Rica with notes on some species. Hydrobiologia 119: 89-99. doi: 10.1007/BF00011948

Cortés J (2012) Marine biodiversity of an Eastern Tropical Pacific oceanic island, Isla del Coco, Costa Rica. Revista de Biología Tropical 60: 131-184.

Denyer P, Cárdenas G (2000) Costas Marinas. In: Denyer P, Kussmaul S (Eds) Geología de Costa Rica. Editorial Tecnológica de Costa Rica, Costa Rica, 185-218.

Dussart BH, Fernando CH (1986) Remarks on two species of copepods of Costa Rica, including a description of a new species of Tropocyclops. Crustaceana 50: 39-44. doi: 10.1163/156854085X00053

Fernández-Álamo MA, Vicencio M (1996) Zooplancton del Domo de Costa Rica: taxonomía y biogeografía. Revista de Biología Tropical 44: 631-642.

Fiedler P (2002) The annual cycle and biological effects of the Costa Rica Dome. Deep Sea Research I 49: 321-338. doi: 10.1016/S0967-0637(01)00057-7

Gavlas AM (2012) Cambios estacionales y relaciones alimentarias de la comunidad planctónica del Embalse Arenal, Costa Rica. M.Sc. thesis, Universidad de Costa Rica, San José, Costa Rica.

Gutiérrez-Aguirre MA, Mercado-Salas NF, Cervantes-Martínez A (2013) Description of Eucyclops tziscao sp. n., E. angeli sp. n., and a new record of E. festivus Lindberg, 1955 (Cyclopoida, Cyclopidae, Eucyclopinae) in Chiapas, Mexico. Zookeys 351: 1-30. doi: $10.3897 /$ zookeys.351.5413

Guzmán H, Obando V (1988) Diversidad y abundancia diaria y estacional del zooplancton marino de la Isla del Caño, Costa Rica. Revista de Biología Tropical 36(1): 139-150.

Haberyan KA, Umaña G, Collado C, Horn SP (1995) Observations on the algae of some Costa Rican lakes. Hydrobiologia 312: 75-85. doi: 10.1007/BF00020763

Huys R, Boxshall GA (1991) Copepod Evolution. The Ray Society, London, 468 pp. 
Mauchline J (1998) The Biology of Calanoid Copepods. Academic Press, England, 710 pp.

Mc Creary JP, Lee HS, Enfield DB (1989) The response of the coastal ocean to strong offshore winds: with application to circulation in the gulfs of Tehuantepec and Papagayo. Journal of Marine Research 47: 81-109. doi: 10.1357/002224089785076343

Mercado-Salas NF, Morales-Suárez E (2014) On Kiefer's American Eucyclops (Copepoda, Eucyclopinae): redescriptions and comments on the historical records of E. delachauxi, E. prionophorus, E. bondi and E. leptacanthus. Zookeys 402: 1-41. doi: 10.3897/zookeys.402.6112

Mercado-Salas NF, Morales-Vela B, Suárez-Morales E, Iliffe TM (2013) Conservation status of the inland aquatic crustaceans in the Yucatan Peninsula, Mexico: shortcomings of a protection strategy. Aquatic Conservation Marine and Freshwater Systems 23(6): 939-951. doi: 10.1002 /aqc. 2350

Mielke W (1992) Six representatives of the Tetragonicipitidae (Copepoda) from Costa Rica. Microfauna Marina 7: 101-146.

Mielke W (1994a) Two co-occurring new Karllangia species (Copepoda: Ameiridae) from the Caribbean coast of Costa Rica. Revista de Biología Tropical 42: 141-153.

Mielke W (1994b) New records of two copepod species (Crustacea) from the Pacific coast of Costa Rica. Microfauna Marina 9: 55-60.

Mielke W (1994c) Microcanuella bisetosa gen.n., sp. n., a new taxon of Canuellidae (Crustacea, Copepoda) from the Costa Rican Pacific coast, with remarks of Galapacanuella beckeri. Bijdragen tot de Dierkunde 64(1): 55-64.

Mielke W (1995) Species of the taxon Schizopera (Copepoda) from the Pacific coast of Costa Rica. Microfauna Marina 10: 89-116.

Molnar JL, Gamboa RL, Revenga C, Spalding MD (2008) Assessing the global threat of invasive species to marine biodiversity. Frontiers in Ecology and the Environment 6: 485-492. doi: 10.1890/070064

Morales-Ramírez A (1987) Caracterización del zooplancton del arrecife en el Parque Nacional Cahuita, Limón, Costa Rica. M.Sc. thesis, Universidad de Costa Rica, San José, Costa Rica.

Morales-Ramírez A (1996) Checklist of copepods from Gulf of Nicoya, Coronado Bay and Golfo Dulce, Pacific coast of Costa Rica, with comments on their distribution. Revista de Biología Tropical 44(3): 103-113.

Morales-Ramírez A (2001) Diversidad marina de Costa Rica: los microcrustáceos: Subclase Copepoda (Crustacea: Maxillopoda). Revista de Biología Tropical 49 (Suppl. 2): 115-133.

Morales-Ramírez A (2008) Caracterización cualitativa del zooplancton del Área de Conservación Marina Isla del Coco (ACMIC), Océano Pacífico de Costa Rica. Revista de Biología Tropical 56(2): 159-169.

Morales-Ramírez A (2011) La diversidad marina del Golfo Dulce, Pacífico sur de Costa Rica: amenazas a su conservación. Biocenosis 24(1/2): 9-20.

Morales-Ramírez A, Murillo MM (1996) Distribution, abundance and composition of coral reef zooplankton, Cahuita National Park, Limón, Costa Rica. Revista de Biología Tropical 44 (2): 619-630.

Morales-Ramirez A, Nowaczyk J (2006) El zooplancton gelatinoso del Golfo Dulce, Pacifico de Costa Rica, durante la transición de la estación lluviosa a la seca 1997-1998. Revista de Biología Tropical 54(1): 201-223. 
Morales-Ramírez A, Suárez-Morales E (2009) Copepods. In: Wehrtmann IS, Cortes J (Eds) Marine Biodiversity of Costa Rica, Central America. Springer, Berlin, 291-305. doi: 10.1007/978-1-4020-8278-8_27

Morales-Ramírez A, Vargas J (1995) Especies comunes de copépodos (Crustacea: Copepoda) pelágicos del Golfo de Nicoya, Costa Rica. Revista de Biología Tropical 43: 207-218.

Pipan T (2005) Epikarst - a Promising Habitat. Copepod fauna, its diversity and ecology: a case study from Slovenia (Europe). ZRC Publishing, Karst Research Institute, $101 \mathrm{pp}$.

Protti M, González V, Freymueller J, Doelger S (2012) Isla del Coco, on Cocos Plate, converges with Isla de San Andrés, on the Caribbean Plate, at $78 \mathrm{~mm} / \mathrm{yr}$. Revista de Biología Tropical 60 (3): 33-41.

Quesada-Alpízar MA, Morales-Ramírez A (2006) Abundancia, composición and distribución vertical del zooplancton no gelatinoso en el Golfo Dulce, costa pacífica de Costa Rica, durante 1997-1998: posible efecto fenómeno de El Niño. Revista de Biología Tropical 54(1): 225-240.

Reid JW (1986) Some usually overlooked cryptic copepod habitats. Syllogeus 58: 594-598.

Reid J (1990a) Continental and coastal free-living Copepoda (Crustacea) from México, Central America and the Caribbean region. In: Navarro D, Robinson JG (Eds) Diversidad Biológica en la Reserva de la Biosfera de Sian Ka'an, Quintana Roo, México. CIQRO/ University of Florida, 175-213.

Reid J (1990b) Canthocampus (Elaphoidella) striblingi, new species (Copepoda: Harpacticoida) from Costa Rica. Proceedings of the Biological Society of Washington 103(2): 336-340.

Rodríguez-Saénz K, Morales-Ramírez A (2012) Composición y distribución del mesozooplancton en una de afloramiento costero (Bahía Culebra, Costa Rica) durante La Niña 1999 y el 2000. Revista de Biología Tropical 60(2): 143-157.

Sibaja-Cordero JA (2012) Composición de la macrofauna bentónica en substratos sedimentarios del Parque Nacional Isla del Coco, Costa Rica. PhD thesis, Universidad de Vigo, Vigo, España.

Silva WM (2008) Diversity and distribution of the free-living freshwater Cyclopoida (Copepoda: Crustacea) in the Neotropics. Brazilian Journal of Biology 68(4): 1099-1106. doi: $10.1590 /$ S1519-69842008000500016

Suárez-Morales E (2003) Historical biogeography and distribution of the freshwater calanoid copepods (Crustacea: Copepoda) of the Yucatan Peninsula, Mexico. Journal of Biogeography 30: 1851-1859. doi: 10.1111/j.1365-2699.2003.00958.x

Suárez-Morales E, Gasca R (1989) Copépodos calanoides epiplanctónicos del Domo de Costa Rica (julio-agosto, 1982). Ciencias Marinas 15: 89-102.

Suárez-Morales E, Gasca R (2012) A new Lepeophtheirus (Copepoda: Siphonomastoida: Caligidae) from Isla del Coco National Park, Costa Rica, Eastern Tropical Pacific. Revista de Biología Tropical 60(3): 235-242.

Suárez-Morales E, Iliffe TM (2005) A new Exumella Fosshagen (Crustacea: Copepoda: Ridgewayiidae) from anchialine waters of the western Caribbean, with comments on regional biogeography. Bulletin of Marine Science 77(3): 409-423.

Suárez-Morales E, Morales-Ramírez A (2001) Nuevo registro de Acartia (Planktacartia) negligens (Copepoda, Calanoida) en el Pacífico Tropical Oriental. Revista de Biología Tropical 44(3): 103-113. 
Suárez-Morales E, Morales-Ramírez A (2003) A new species of Cymbasoma (Copepoda: Monstrilloida) from the Pacific coast of Costa Rica, Central America. Proceedings of the Biological Society of Washington 116: 206-214.

Suárez-Morales E, Morales-Ramírez A (2009) New species of Monstrilloida (Crustacea, Copepoda) from the Eastern Tropical Pacific. Journal of Natural History 43: 1257-1271. doi: 10.1080/00222930902894419

Suárez-Morales E, Carrillo A, Morales-Ramírez A (2013) Report on some Monstrilloids (Crustacea, Copepoda) from a reef area off the Caribbean coast of Costa Rica, Central America with descriptions of two new species. Journal of Natural History 47: 619-638. doi: $10.1080 / 00222933.2012 .742933$

Suárez-Morales E, Mercado-Salas NF, Morales-Ramírez A (2011) Morphological variability and distribution of the exotic Asian Mesocyclops thermocyclopoides (Copepoda: Cyclopoida) in the Neotropical region. Zoología 28(5): 673-679. doi: 10.1590/S198446702011000500016

Suárez-Morales E, Reid JW, Elías-Gutiérrez M (2005) Diversity and distributional patterns of Neotropical freshwater Copepods (Calanoida: Diaptomidae). International Review of Hydrobiology 90: 71-83. doi: 10.1002/iroh.200410742

Suárez-Morales E, Reid JW, Gasca R (2000) Free-living marine and freshwater Copepoda (Crustacea) from Mexico. In: Llorente-Bousquets J, González-Soriano E, Papavero N (Eds) Biodiversidad, Taxónoía y Biogeografía de Artrópodos de México. Hacia una Síntesis de su Conocimiento. Vol. II. CONABIO/UNAM, México, 171-190.

Svendsen H, Rosland R, Myking S, Vargas JA, Lizano OG, Alfaro EJ (2006) A physical-oceanographic study of Golfo Dulce, Costa Rica. Revista de Biología Tropical 54(1): 147-170.

Umaña G, Collado C (1990) Asociación planctónica en el Embalse Arenal, Costa Rica. Revista de Biología Tropical 38: 311-321.

Vargas JA (1995) The Gulf of Nicoya estuary, Costa Rica: past, present and future cooperative Research. Helgoländer Meeresuntersuchungen 49: 821-828. doi: 10.1007/BF02368405

Wehrtmann IS, Cortés J (2009) Marine Biodiversity of Costa Rica, Central America. Springer, Berlin, 538 pp. doi: 10.1007/978-1-4020-8278-8 